\title{
Role of $\alpha$-Actinin 2 in Cytoadherence and Cytotoxicity of Trichomonas vaginalis
}

\author{
Hye-Yeon Lee, Juri Kim, and Soon-Jung Park* \\ Department of Environmental Medical Biology and Institute of Tropical Medicine, Brain Korea 21 PLUS Project for Medical Science, Yonsei \\ University College of Medicine, Seoul 03722, Republic of Korea
}

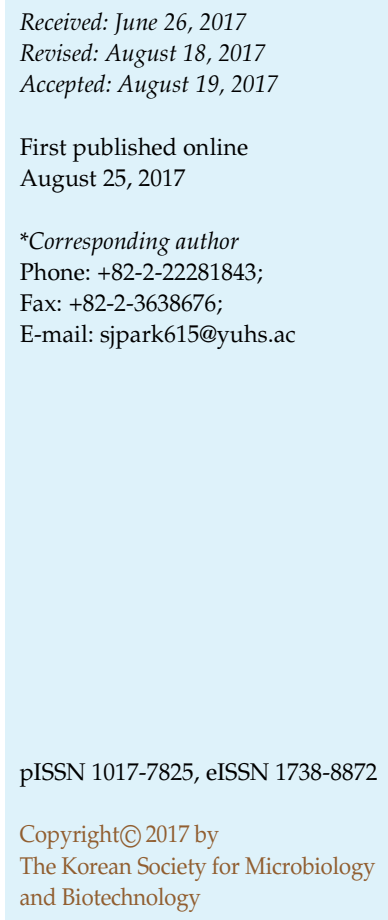

Trichomonas vaginalis is a pathogen that triggers severe immune responses in hosts. T. vaginalis $\alpha$-actinin 2 (Tv $\alpha$-actinin 2) has been used to diagnose trichomoniasis. Tv $\alpha$-actinin 2 was dissected into three parts; the N-terminal, central, and C-terminal portions of the protein (\#1, $\# 2$, and \#3, respectively). Western blot of these Tv $\alpha$-actinin 2 proteins with pooled patients' sera indicated that \#2 and \#3, but not \#1, reacted with those sera. Immunofluorescence assays of two different forms of T. vaginalis (trophozoites and amoeboid forms), using anti-Tvaactinin 2 antibodies, showed localization of Tv $\alpha$-actinin 2 close to the plasma membranes of the amoeboid form. Fractionation experiments indicated the presence of Tva-actinin 2 in cytoplasmic, membrane, and secreted proteins of T. vaginalis. Binding of fluorescence-labeled Trichomonas to vaginal epithelial cells and prostate cells was decreased in the antibody blocking experiment using anti-Tv $\alpha$-actinin 2 antibodies. Pretreatment of T. vaginalis with anti-rTv $\alpha-$ actinin 2 antibodies also resulted in reduction in its cytotoxicity. Flow cytometry, ligandbinding immunoblotting assay, and observation by fluorescence microscopy were used to detect the binding of recombinant Tv $\alpha$-actinin 2 to human epithelial cell lines. Specifically, the truncated N-terminal portion of Tv $\alpha$-actinin 2, Tv $\alpha$-actinin $2 \# 1$, was shown to bind directly to vaginal epithelial cells. These data suggest that $\alpha$-actinin 2 is one of the virulence factors responsible for the pathogenesis of $T$. vaginalis by serving as an adhesin to the host cells.

Keywords: Trichomonas vaginalis, $\alpha$-actinin 2 , cytoadherence, cytotoxicity

\section{Introduction}

Trichomonas vaginalis is known to be an important pathogen causing the most prevalent non-viral sexually transmitted disease worldwide and has been used for the study of host-parasite co-rrelationship [1]. Host immunogens and protozoan factors cause a distinct interaction during $T$. vaginalis infection in the vaginal tract. There are a few studies about the host-parasite co-rrelationship, especially trophozoites of T. vaginalis interacting with mucin [2], and then contact with vaginal epithelial cells (VECs) at ironregulated surface proteins of T. vaginalis [3]. Adherence of the host epithelium by $T$. vaginalis also occurs via a specific interaction with the extracellular matrix basement membrane glycoproteins [4].

In the pathogenesis of $T$. vaginalis, iron is one of the virulence factors that modulates diverse aspects of T. vaginalis, including cytoadherence, metabolic activity, and resistance to complement lysis $[5,6]$. T. vaginalis has ways of acquiring iron from lactoferrin [3]. T. vaginalis takes up lactoferrin via specific receptors, which trigger iron accumulation and activity of pyruvate ferridoxin oxidoreductase [7]. Iron availability from lactoferrin caused enhanced binding to the host epithelial cells by increased amount of adhesins [8]. T. vaginalis also interacts with erythrocytes, which provide both lipid and iron for the parasite [9], and the action of two adhesion proteins, AP51 and AP65, triggers iron acquisition from hemoglobin by T. vaginalis [10]. Cultivation of T. vaginalis in iron-depleted medium shows no pathology, but could cause subcutaneous abscesses in mice [11].

Adhesion of fluorescence-labeled T. vaginalis to various 
host cells has been observed by fluorescence microscopy [12]. Studies using erythrocytes as target cells demonstrated that hemolysis by $T$. vaginalis is mediated by perforins and the cytoskeleton [13]. Iron-regulated surface proteins of T. vaginalis (AP65, AP51, AP33, and AP23) have been postulated to play essential roles in the adherence of T. vaginalis to VECs [3]. Interestingly, a mutant $T$. vaginalis lacking its lipoglycan, TvLG, indicated that this surface molecule also plays a role in attachment of the parasite to host epithelial cells via specific interactions with galectin-1 of the host cell [14]. An evaluation of the adherence and cytotoxicity of $26 T$. vaginalis strains to prostate and ectocervical epithelia, as well as erythrocytes, demonstrated variable pathogenicity among different $T$. vagnialis strains, but all strains showed contact-dependent cytolysis [15].

Among the immunogenic proteins of $T$. vaginalis identified in the previous investigation [16], T. vaginalis $\alpha$-actinin 2 (Tvo-actinin 2) had further been studied, showing its ability to modulate host immune responses, using mouse dendritic cells as well as human vaginal and prostate epithelial cells [17]. Higher serological responses against Tvo-actinin 2 in prostate cancer cases compared with the control cases indicated a relationship between antiT. vaginalis seropositivity and risk for prostate cancer in men [18, 19]. Reactive epitopes of $\alpha$-actinin 2 have been finely mapped using sera from both male and female trichomoniasis patients [20]. Using a custom spot membrane loaded with overlapped 11-mer peptides covering Tv $\alpha$ actinin 2, 13 epitopes were found to be scattered in $\alpha$-actinin 2 when tested against women's sera, whereas only 4 of the 15 epitopes in the C-terminal portion of $\alpha$-actinin 2 were reactive to men's sera.

In this study, the role of Tv $\alpha$-actinin 2 in parasite adherence to the prostate cell line RWPE- 1 and to VECs was examined by direct binding assays, and the domain of Tv $\alpha$-actinin 2 responsible for cytoadherence was also defined.

\section{Materials and Methods}

\section{Parasite and In Vitro Transformation}

T. vaginalis KT4 [21] was grown in Diamond's Trypticase Yeast extract Maltose (TYM) medium [22] with 10\% horse serum (Gibco $\mathrm{BRL}$, Germany) at $37^{\circ} \mathrm{C}$ in $5 \% \mathrm{CO}_{2}$, and transferred into fresh medium daily. T. vaginalis trophozoites were converted into the amoeboid form by incubating them in fibronectin-coated plates (3 $\mu \mathrm{g} / \mathrm{ml})$ for $30 \mathrm{~min}$.

\section{Bacterial and Human Cell Cultures}

Plasmids used for molecular manipulations are listed in Table 1. Escherichia coli BL21 (DE3) cells were grown at $37^{\circ} \mathrm{C}$ in Luria-Bertani medium $(0.5 \%$ yeast extract, $1 \%$ tryptone, $1 \% \mathrm{NaCl}$, $\mathrm{pH}$ 7.5) supplemented with $100 \mu \mathrm{g} / \mathrm{ml}$ ampicillin (Sigma-Aldrich, USA) in order to maintain the plasmids. Medium components were obtained from BD-Difco (USA).

Human VECs (ATCC CRL-2614; American Type Culture Collection, USA) were cultivated in Dulbecco's modified Eagle's medium (DMEM; Gibco BRL) supplemented with 10\% heatinactivated fetal bovine serum (FBS), penicillin $(100 \mathrm{U} / \mathrm{ml})$, and streptomycin $(100 \mu \mathrm{g} / \mathrm{ml})$. Human prostatic epithelial cells (RWPE-1, ATCC CRL-11609; American Type Culture Collection) were grown using Keratinocyte Serum Free Medium (K-SFM; Gibco BRL) containing epidermal growth factor $(0.2 \mathrm{ng} / \mathrm{ml})$, bovine pituitary extract $(0.05 \mathrm{mg} / \mathrm{ml})$, FBS $(5 \%)$, penicillin $(100 \mathrm{U} / \mathrm{ml})$, and streptomycin $(100 \mu \mathrm{g} / \mathrm{ml})$. These human epithelial cells were

Table 1. Primers and plasmids used in this study.

\begin{tabular}{llc}
\hline Name & \multicolumn{1}{c}{ Relevant characteristics $^{\mathrm{a}}$} & Source \\
\hline Primers & & \\
$\alpha$-actinin2-1F & CGGAATTCGATGTCTGTTCGTCGTGAAGG (EcoRI) \\
$\alpha$-actinin2-1R & CGGCTCGAGTGCGAAAGCCTTCTCGCAGGCG (XhoI) & \\
$\alpha$-actinin2-2F & ACGCGTCGACGCTTGCAAGGAACTCGGCAT (SalI) & \\
$\alpha$-actinin2-2R & TTTTTTGCGGCCGCCTTGTAGTTGAGCTTGAATT (NotI) & Novagen \\
$\alpha$-actinin2-3F & ACGCGTCGACGTCACATACACATACTCTGA (SalI) & [30] \\
$\alpha$-actinin2-3R & TTTTTTGCGGCCGCTTGGTTGTAAAGTGAGT (NotI) & This study \\
Plasmids & & This study \\
pET21b & Expression vector for a histidine-tagged protein & This study \\
pET $\alpha$-actinin2 & pET21b, 2,786-bp $\alpha$-actinin 2 gene & \\
pETact1 & pET21b, 591-bp N-terminal portion of $\alpha$-actinin 2 gene \\
pETact2 & pET21b, 1,358-bp central portion of -actinin 2 gene & \\
pETact3 & pET21b, 843-bp C-terminal portion of $\alpha$-actinin 2 gene & \\
\hline
\end{tabular}

${ }^{a}$ Underlined sequences indicate restriction sites. 


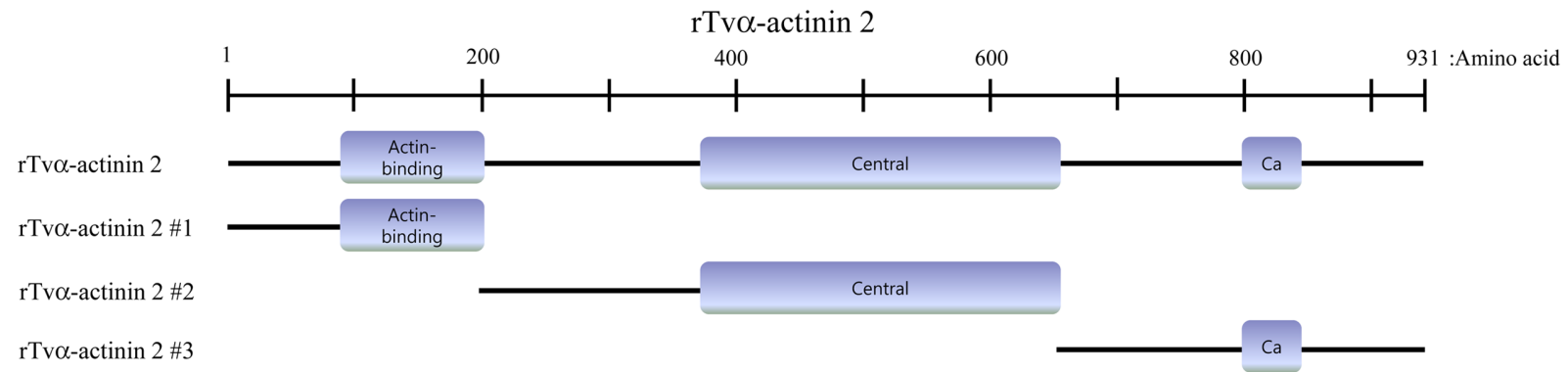

Fig. 1. $r$ Tv $\alpha$-actinin 2 protein of T. vaginalis and its truncated derivatives used in this study.

Four different $\mathrm{rTv} \alpha$-actinin 2 polypeptides were prepared containing the complete Tv $\alpha$-actinin 2 open reading frame (rTv $\alpha$-actinin 2), and the Nterminal (rTv $\alpha$-actinin $2 \# 1$ ), central (rTv $\alpha$-actinin $2 \# 2)$, and C-terminal (rTv $\alpha$-actinin 2 \#3) regions. The three boxes above these numbers represent the actin-binding domain, central antigenic region, and $\mathrm{Ca}^{2+}$-binding domain of Tv $\alpha$-actinin 2 (Addis et al. [25]).

cultured to $80-90 \%$ confluence for use in the adhesion and cytotoxicity assays at $37^{\circ} \mathrm{C} / 5 \% \mathrm{CO}_{2}$.

\section{Expression and Purification of Recombinant Tv $\alpha$-Actinin 2 Polypeptides}

The DNA fragment encoding Tv $\alpha$-actinin 2 was divided into three portions (Fig. 1). The $5^{\prime}$-region of the Tv $\alpha$-actinin 2 gene (591 bp) was made from the genome of T. vaginalis KT4 by PCR using the primers $\alpha$-actinin $2-1 \mathrm{~F}$ and $\alpha$-actinin2-1R (Table 1 ), and the central region of the Tv $\alpha$-actinin 2 gene $(1,358 \mathrm{bp})$ was amplified with another set of primers, $\alpha$-actinin $2-2 \mathrm{~F}$ and $\alpha$-actinin2-2R. The 3 '-region of the Tv $\alpha$-actinin 2 gene was amplified as an 843-bp DNA fragment, using the primers $\alpha$-actinin2-3F and $\alpha$-actinin2-3R. Each of the resulting Tv $\alpha$-actinin 2 DNA fragments was cloned into pET21b (Novagen, Germany), yielding overexpression plasmids for the truncated $\alpha$-actinin 2 proteins (pETact1, pETact2, and pETact3).

These truncated Tv $\alpha$-actinin 2 proteins were expressed as histidine-tagged form in E. coli BL21 (DE3) with $1 \mathrm{mM}$ isopropyl $\beta$-D-thiogalactoside, and then purified using nickel-nitrilotriacetic acid affinity chromatography as described by the manufacturer (Qiagen, Germany). Full-length recombinant Tv $\alpha$-actinin 2 (rTv $\alpha$ actinin 2) was prepared as previously described [17].

\section{Western Blot Analysis}

These $r$ Tv $\alpha$-actinin 2 polypeptides (100 ng) were subjected to sodium dodecyl sulfate-polyacrylamide gel electrophoresis (SDSPAGE), and then transferred to a nitrocellulose filter (Millipore, USA). The membrane was incubated with pooled sera (1:200 dilution) derived from trichomoniasis patients in a blocking solution (Tris-buffered saline (TBS; $50 \mathrm{mM}$ Tris-Cl and $150 \mathrm{mM}$ $\mathrm{NaCl}, \mathrm{pH} 7.5), 5 \%$ skim milk, and $0.05 \%$ Tween 20 ), and incubated with alkaline phosphatase (AP)-conjugated anti-human IgG (Sigma-Aldrich). As a control, these proteins were also reacted with normal sera (1:200 dilution) derived from uninfected individuals. The immunoreactive protein was visualized using the nitroblue tetrazolium/5-bromo-4-chloro-3-indolyl phosphate system (Promega, USA).

\section{Immunofluorescence Assays (IFA)}

Trophozoites and amoeboid forms of $T$. vaginalis were adhered to glass slides, and then incubated with $100 \%$ methanol at $-20^{\circ} \mathrm{C}$ for $10 \mathrm{~min}$. After $1 \mathrm{~h}$ incubation in a blocking buffer (phosphatebuffered saline (PBS: $1.7 \mathrm{mM} \mathrm{KCl}, 137 \mathrm{mM} \mathrm{NaCl}, 2 \mathrm{mM} \mathrm{KH}_{2} \mathrm{PO}_{4}$, and $\left.10 \mathrm{mM} \mathrm{Na}_{2} \mathrm{HPO}_{4}, \mathrm{pH} 7.3\right), 5 \%$ goat serum, and $3 \%$ bovine serum albumin (BSA)), the cells were reacted with anti-Tv $\alpha$ actinin 2 polyclonal antibodies (1:800 dilution) [17] at $4^{\circ} \mathrm{C}$. Following three washes with PBS, the cells were reacted with Alexa-Fluora 555-conjugated anti-rat IgG (1:400 dilution; Life Technologies, USA) at $37^{\circ} \mathrm{C}$ for $2 \mathrm{~h}$. The slides were washed with PBS and mounted with $1.5 \mu \mathrm{g} / \mathrm{ml} 4^{\prime} 6$-diamidino-2-phenylindole (DAPI; Sigma-Aldrich) anti-fade mounting medium (VECTASHIELD; Vector Laboratories, USA). The cells were then observed under an Axiovert 200 fluorescent microscope or LSM710 confocal microscopy (Carl Zeiss, Germany).

\section{Protein Fractionation from Amoeboid Form of T. vaginalis}

Cytoplasmic and membrane fractions of the T. vaginalis were prepared using the subcellular Proteome Extraction Kit (Merck, Germany). One microgram of protein samples was separated by $10 \%$ SDS-PAGE and transferred to polyvinylidene difluoride (PVDF) membranes and blocked with the blocking solution for $1 \mathrm{~h}$ at room temperature. The membranes were treated with anti-Tv $\alpha-$ actinin 2 antibodies at $4^{\circ} \mathrm{C}$ overnight, and reacted with horseradish peroxidase (HRP)-conjugated secondary antibodies (Cell Signaling, USA). An Enhanced Chemiluminescence System (ECL; GE Healthcare Life Science, UK) was used for signal detection.

\section{Labeling of T. vaginalis and Blocking Experiments}

T. vaginalis cells were stained with the fluorescent Cell Tracker Orange CMTMR dye (Invitrogen, USA). Briefly, T. vaginalis cells $\left(1 \times 10^{6}\right.$ cells $\left./ \mathrm{ml}\right)$ were incubated in pre-warmed cell tracker dye solution $(0.5 \mu \mathrm{M}$ in TYM medium without serum) for $30 \mathrm{~min}$ at $37^{\circ} \mathrm{C}$. The cells were harvested by centrifugation, and then incubated in TYM medium supplemented with $10 \%$ FBS at $37^{\circ} \mathrm{C}$ for $30 \mathrm{~min}$. The parasites were then washed twice: once with TYM medium and once with complete K-SFM. 
Prior to cytoadherence assays, T. vaginalis trophozoites $\left(1 \times 10^{8}\right)$ were pre-incubated with anti-Tv $\alpha$-actinin 2 antibodies for $1 \mathrm{~h}$ at a concentration of $30 \mu \mathrm{g} / \mathrm{ml}$. As a control, pre-immune rat serum was used to monitor the nonspecific effect of serum on the cytoadherence of $T$. vaginalis to the host cells.

\section{Cytotoxicity Assays and Blocking Experiments}

VECs $\left(3 \times 10^{4}\right.$ cells/well) inoculated into 96-well plates were cultured to confluence at $37^{\circ} \mathrm{C} / 5 \% \mathrm{CO}_{2} . T$. vaginalis cells $\left(3 \times 10^{5}\right.$ cells/well) at log phase were added to the confluent VECs, and then incubated for $4 \mathrm{~h}$ at $37^{\circ} \mathrm{C} / 5 \% \mathrm{CO}_{2}$. Lysis of VECs was measured by determining the level of released lactate dehydrogenase (LDH) using the Cytotox ${ }^{96}$ Non-Radioactive Cytotoxicity Kit (Promega). The level of released LDH was determined as follows: $\left[\left(\mathrm{OD}_{490}\right.\right.$ of experimental $\mathrm{LDH}-\mathrm{OD}_{490}$ of spontaneous $\left.\mathrm{LDH}\right) /\left(\mathrm{OD}_{490}\right.$ of maximum $\mathrm{LDH}-\mathrm{OD}_{490}$ of spontaneous $\left.\left.\mathrm{LDH}\right)\right] \times 100$. The spontaneous LDH was the activity of LDH released from untreated VECs, whereas the maximum LDH was the LDH activity caused from complete lysis of VECs due to Triton X-100 treatment.

To examine the role of $\mathrm{Tv} \alpha$-actinin 2 in cytotoxicity of $T$. vaginalis to VECs, T. vaginalis trophozoites $\left(1 \times 10^{8}\right)$ were pre-incubated with anti-Tv $\alpha$-actinin 2 antibodies for $1 \mathrm{~h}$ at a concentration of $30 \mu \mathrm{g} / \mathrm{ml}$. In order to monitor the nonspecific effect of serum on the cytotoxicity of T. vaginalis to VECs, pre-immune rat serum was used as a control.

\section{Labeling of rTv $\alpha$-Actinin 2}

To estimate the binding of rTv $\alpha$-actinin 2 to the host cells, Tv $\alpha$ actinin 2 proteins were labeled with a fluorescent probe using an Alexa-Fluor 555 Microscale Protein Labeling Kit (A30007; Molecular Probes, USA). One hundred microgram of recombinant polypeptides was reacted with Alexa-Fluor 555 succinimidyl ester, and then passed through the spin-filters supplied with the kit to remove unincorporated probe. Fluorescence of the proteins was measured at 280 and $555 \mathrm{~nm}$ in order to check labeling of the protein as directed by the manufacturer. Alexa-Fluor 555-labeled $\alpha$-actinin $2(100 \mu \mathrm{g} / \mathrm{ml})$ was stored at $-20^{\circ} \mathrm{C}$ before being used for the binding assays.

\section{Fluorescence-Activated Cell Sorter (FACS) Analysis}

FACS analysis was carried out by using a BD FACS Calibur flow cytometer (BD Biosciences, USA). For the adhesion assay, Cell Tracker Orange CMTMR-stained T. vaginalis can be detected in excitation and emission spectrum peak wavelengths of approximately 488 and $565 \mathrm{~nm}$. Additionally, Alexa-fluor 555labeled proteins have fluorescence excitation and emission maxima of 555 and $565 \mathrm{~nm}$. To detect CMTMR-stained parasites or Alexa-fluor 555 dye-labeled proteins in the presence of VECs or RWPE-1, voltage sets were made based on the auto-fluorescent signal of the VECs and RWPE-1. Finally, FACS data analyses were performed using FlowJo software (FlowJo LLC, USA).

\section{Fluorescence Microscopy}

rTv $\alpha$-actinin 2 proteins were conjugated with a fluorescent probe using an Alexa-Fluor 555 Microscale Protein Labeling Kit as described above. VEC cells $\left(2 \times 10^{4}\right.$ cells per well) were grown on glass coverslips in 24-well plates for $24 \mathrm{~h}$. The cells were incubated with $3 \%$ BSA for $30 \mathrm{~min}$ in DMEM without serum followed by a $1 \mathrm{~h}$ treatment with $10 \mu \mathrm{g} / \mathrm{ml}$ Alexa-Fluor 555-labeled polypeptides at $37^{\circ} \mathrm{C}$, and then fixed in $3.7 \%$ paraformaldehyde/ PBS at room temperature for $30 \mathrm{~min}$. After being washed with PBS three times, the cells were observed under an Axiovert 200 fluorescence microscope.

\section{Ligand-Binding Immunoblotting Assay}

Each well of a 24-well culture plate was seeded with VECs $(2 \times$ $10^{5}$ ), and incubated overnight at $37^{\circ} \mathrm{C}$ in the presence of $5 \% \mathrm{CO}_{2}$. After removing the medium and washing twice with DMEM, the cells were incubated in $1 \mathrm{ml}$ of serum-free DMEM containing $10 \mu \mathrm{g}$ of the $\mathrm{rTv} \alpha$-actinin 2 proteins as previously described [23]. After five washes with DMEM, the cells were disrupted with $150 \mu \mathrm{l}$ of lysis buffer (10 mM Tris- $\mathrm{HCl}, \mathrm{pH} 8.4$, and $0.8 \%$ SDS) and boiled for $10 \mathrm{~min}$. To examine whether rTv $\alpha$-actinin 2 was stably bound to VEC monolayers, a portion of the lysate (15 $\mu \mathrm{g}$ of protein) was subjected to SDS-PAGE and then transferred onto a PVDF membrane. The membranes were blocked with 5\% non-fat dry milk in TBS with $0.05 \%$ Tween 20 for $1 \mathrm{~h}$ at room temperature, treated with anti-Tv $\alpha$-actinin 2 antibodies at $4^{\circ} \mathrm{C}$ overnight, and subsequently reacted with AP-conjugated anti-rat IgG (Sigma-Aldrich).

In the case of truncated $\mathrm{rTv} \alpha$-actinin 2 proteins, \#1, \#2, and \#3, the membranes were treated with anti-histidine Abs (IG Therapy, Korea) instead of anti-rTv $\alpha$-actinin 2 antibodies. They were then incubated with HRP-conjugated secondary antibodies (Cell Signaling), and the immunoreactive bands were visualized using an ECL System (GE Healthcare Life Science). To monitor the protein level of VECs in each treatment, the VEC lysates were monitored for their $\beta$-actin levels using anti-human $\beta$-actin antibodies (Millipore).

\section{Statistical Analyses}

Results are indicated as the means \pm standard deviations obtained from three independent experiments. Data were analyzed by pairwise comparison using the Student's $t$-test (SYSTAT program, SigmaPlot ver. 10; Systat Software Inc., USA). Differences were considered statistically meaningful if the $p$-values were $<0.05$.

\section{Results}

\section{Reactivity of Recombinant Tv $\alpha$-Actinin 2 Proteins against Patients' Sera}

T. vaginalis $\alpha$-actinin 2 was previously identified as one of the immunogens recognized by sera of $T$. vaginalisinfected women [24]. Full-length rTv $\alpha$-actinin 2 protein was expressed in E. coli and purified via $\mathrm{Ni}^{2+}$-NTA affinity chromatography. ELISA was performed with 10 female patients' sera (data not shown). Nine of 10 sera were reactive 


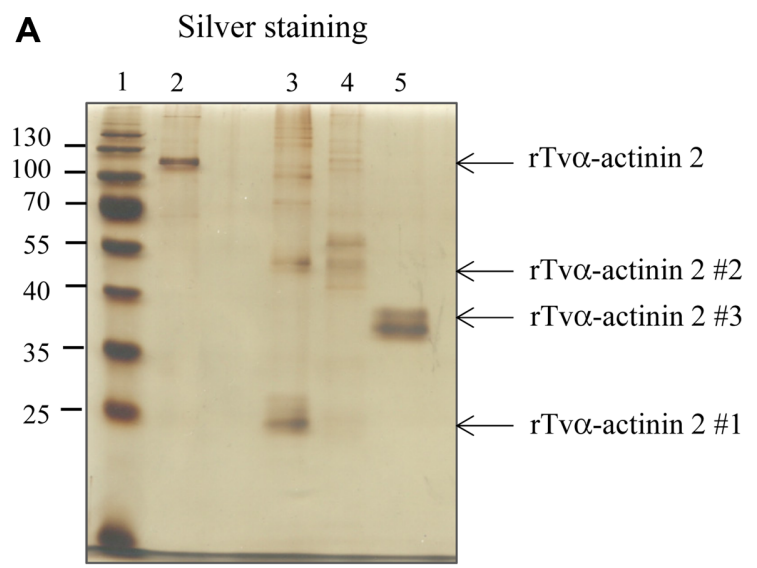

B

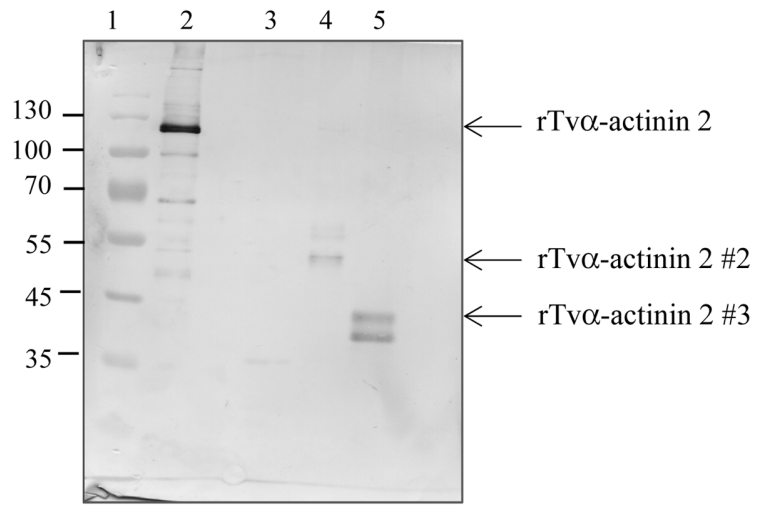

C Pre-immune sera

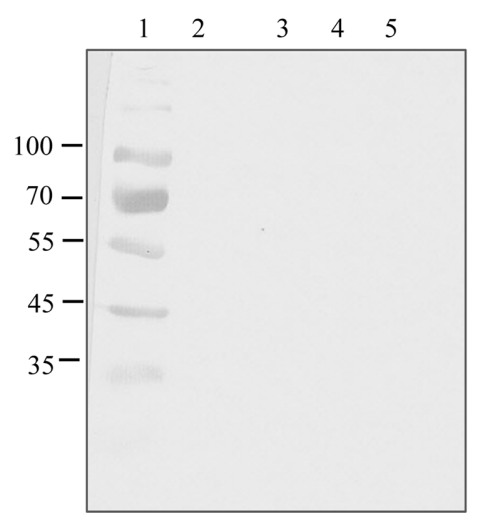

Fig. 2. Reactivity of $r T v \alpha$-actinin 2 polypeptides to a pool of immunopositive sera.

(A) A silver-stained SDS-PAGE gel of $r T v \alpha$-actinin 2 proteins. (B) Western blot analysis of these Tv $\alpha$-actinin 2 proteins with sera derived from patients with trichomoniasis. (C) Western blot analysis of these Tv $\alpha$-actinin 2 proteins with a negative control serum showing no reactivity to $\mathrm{rTv} \alpha$-actinin 2 by ELISA. Lane1: protein size marker; lane 2: full-length $r T v \alpha$-actinin 2; lane 3: $r T v \alpha$-actinin $2 \# 1$; lane 4: rTv $\alpha$-actinin 2 \#2; lane 5: rTv $\alpha$-actinin $2 \# 3$. to this recombinant protein. In the subsequent experiment, a pool of nine sera was used for immunopositive sera, and the human serum showing no reactivity to $r T v \alpha$-actinin 2 served as a negative control (Fig. 2).

Analysis of the deduced amino acid sequence of $\operatorname{Tv} \alpha-$ actinin 2 revealed that this protein is composed of an $\mathrm{N}$-terminal actin-binding domain, a central domain rich in $\alpha$-helices, and a C-terminal region with $\mathrm{Ca}^{2+}$-binding activity [25]. Three truncated recombinant Tv $\alpha$-actinin 2 proteins were prepared (Fig. 2A) and used to define the protein regions reactive to patients' sera. Western blot analysis of these partial $\mathrm{rTv} \alpha$-actinin 2 proteins with the pooled patients' sera indicated that the central (\#2) and C-terminal portions (\#3), but not the N-terminal part (\#1) of Tva-actinin 2, reacted with patients' sera (Fig. 2B). In contrast, these proteins did not show any reactivity with the pre-immune serum used as a negative control (Fig. 2C).

\section{Localization of Tv $\alpha$-Actinin 2 in T. vaginalis}

Using antibodies specific to rTva-actinin 2, IFAs were performed to examine the intracellular location of $\operatorname{Tv} \alpha-$ actinin 2 in two different forms of $T$. vaginalis, trophozoites and amoebic form (Figs. 3A and 3B/3C, respectively). Tv $\alpha$ actinin 2 was mainly detected in the cytoplasm of $T$. vaginalis trophozoites. A conformational change into the amoeboid form occurred upon contact of $T$. vaginalis trophozoites with fibronectin. Interestingly, the amoebic form of T. vaginalis showed localization of Tva-actinin 2 near the plasma membranes. Observation of the amoebic T. vaginalis at higher magnification indicated that Tv $\alpha$-actinin 2 also locates near the membranes.

In the subsequent experiment, fibronectin-induced amoebic T. vaginalis cells were fractionated into cytoplasmic, membrane, and secreted proteins, and then analyzed by western blot analysis using anti-Tv $\alpha$-actinin 2 antibodies (Fig. 3D). Interestingly, this protein was found in all the three fractions of T. vaginalis, indicating that Tv $\alpha$-actinin 2 is secreted or present at the surface of T. vaginalis.

Additional IFAs were performed to observe the location of Tva-actinin 2 when $T$. vaginalis was incubated with VECs or RWPE-1 (Figs. 3E and 3F, respectively). Most T. vaginalis cells were in the amoeboid form upon incubation with both the vaginal and prostate epithelial cell lines and, under these conditions, IFAs of T. vaginalis with anti-rTv $\alpha$ actinin 2 antibodies showed localization of Tva-actinin 2 near its plasma membrane.

\section{Binding of T. vaginalis to VEC and RWPE-1 Cells}

The ability of the pathogenic trichomonads to adhere to 
A

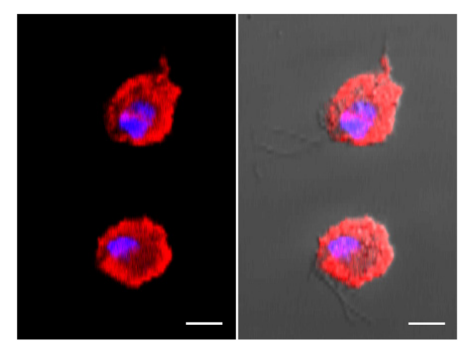

D

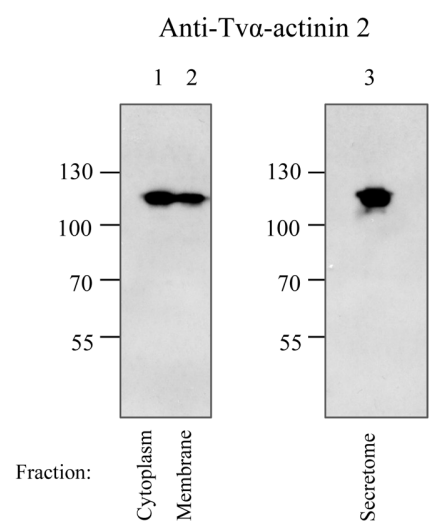

B

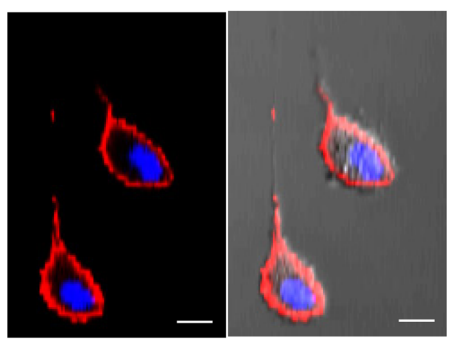

C

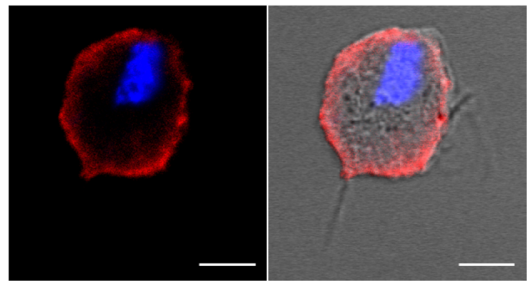

E

Amoeboid form with VECs
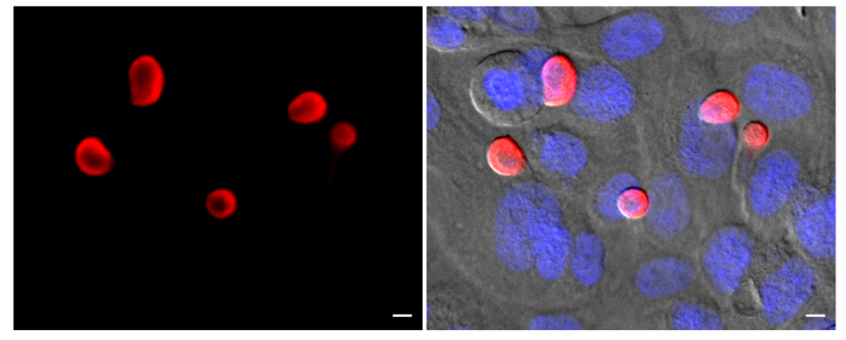

F

Amoeboid form with RWPE-1
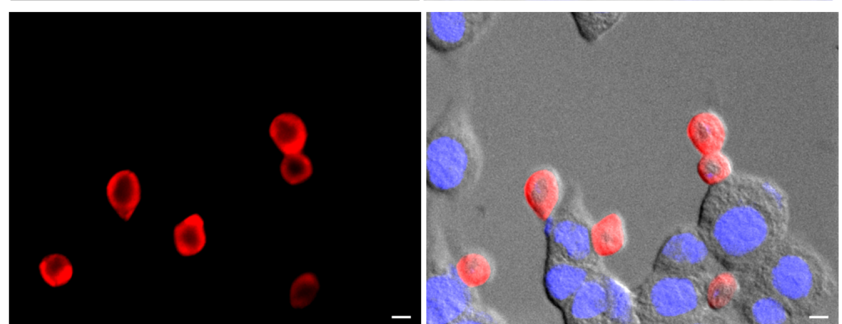

Fig. 3. Localization of Tv $\alpha$-actinin 2 in T. vaginalis.

Using $r \mathrm{Tv} \alpha$-actinin 2 antibodies, the localization of Tv $\alpha$-actinin 2 was examined in T. vaginalis trophozoites (A), amoeboid forms (B, C), T. vaginalis incubated with vaginal epithelial cells (VECs) (E), and T. vaginalis incubated with prostate cells (RWPE-1) (F). The scale bars represent $5 \mu \mathrm{m}$. (D) T. vaginalis exposed to fibronectin was used for the fractionation experiments into cytoplasmic, membrane, and secreted proteins. The protein fractions were analyzed by western blot assay using anti-Tv $\alpha$-actinin 2 antibodies.

host cells is required for its survival and a prerequisite to host cell killing [26]. Here, adherence of T. vaginalis to VEC or RWPE-1 was quantified by flow cytometry analysis using fluorescence-labeled T. vaginalis (Figs. 4A and 4B for VEC; Fig. 4C for RWPE-1).

An experiment was then performed to examine whether masking of Tv $\alpha$-actinin 2 on the T. vaginalis surface inhibited the parasites' adherence to these cells (Fig. 4B for VEC; Fig. 4C for RWPE-1). For both cell lines, a significant decrease in cytoadherence of $T$. vaginalis was observed upon pretreatment with anti-Tvo-actinin 2 antibodies. As a control, T. vaginalis incubated with pre-immune serum was used for the adherence tests, and no effect on VEC or RWPE-1 binding ability was observed.

\section{Role of Tv $\alpha$-Actinin 2 in Cytotoxicity of T. vaginalis to VECs}

We examined whether incubation of $T$. vaginalis with anti-Tva-actinin 2 antibodies affected the pathogen's ability to kill VECs. After VECs were incubated with T. vaginalis or T. vaginalis pretreated with anti-Tv $\alpha$-actinin 2 antibodies, the viability of the VECs was monitored by measuring the released LDH (Fig. 4D). VECs treated with T. vaginalis at a multiplicity of infection of 10 for $4 \mathrm{~h}$ demonstrated $82 \%$ $\mathrm{LDH}$ release relative to the $\mathrm{LDH}$ of the total cells used in the assays. The cytotoxicity of T. vaginalis pretreated with anti-Tv $\alpha$-actinin 2 antibodies was decreased to $59 \%$ in contrast with $80 \%$ cytotoxicity of pre-immune serumtreated T. vaginalis.

\section{Binding of Tv $\alpha$-Actinin 2 to VECs and RWPE-1 Cell Lines}

We then investigated whether Tv $\alpha$-actinin 2 binds directly to host cells, by (i) flow cytometry analysis, (ii) observation of the cell lines incubated with fluorescence-labeled rTv $\alpha$ actinin 2 proteins, and (iii) ligand immunoblotting binding assays.

After VEC and RWPE-1 cells were incubated with 


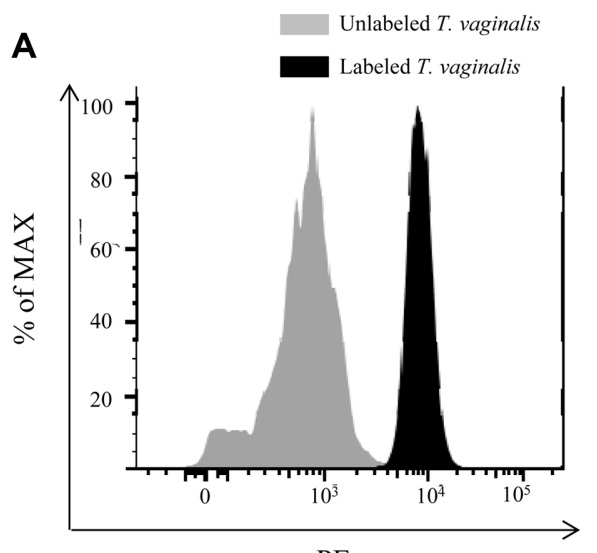

PE

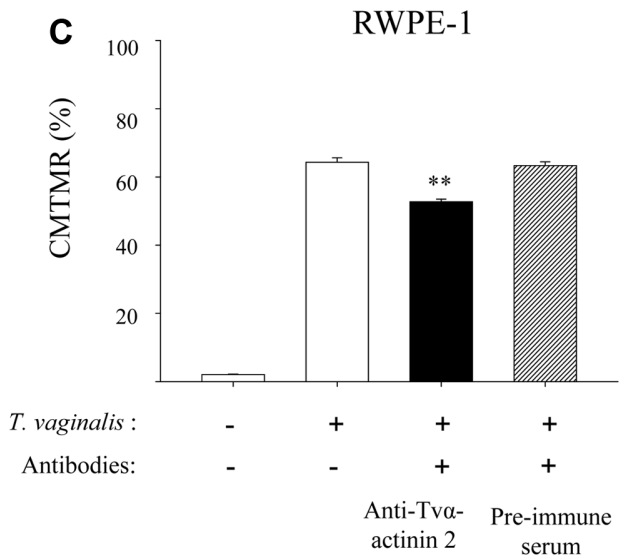

B

VECs

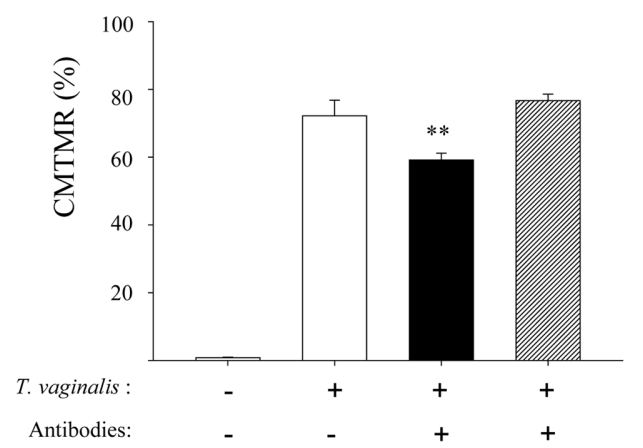

Anti-Tv $\alpha$ - Pre-immune actinin 2 serum

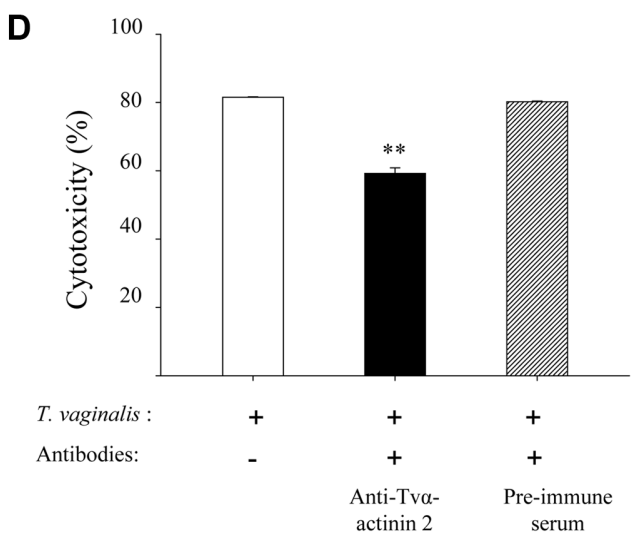

Fig. 4. Role of Tv $\alpha$-actinin 2 in T. vaginalis cytoadherence and cytotoxicity to vaginal epithelial cells (VECs) and prostate epithelial (RWPE1) cells.

(A) A histogram showing the binding of fluorescence-labeled T. vaginalis to VECs determined by flow cytometric analysis. T. vaginalis was stained with $0.5 \mu \mathrm{M}$ CMTMR to determine staining intensity compared with unstained T. vaginalis. Stained trichomonads were incubated with VECs at an MOI of 10 for $6 \mathrm{~h}$ and washed in free growth medium five times to discriminate unbound cells. (B, C) Bar graphs that show average adherence of CMTMR-stained T. vaginalis or CMTMR-stained T. vaginalis with VECs (B) and RWPE-1 (C) pretreated with anti-Tv $\alpha$-actinin 2 antibodies for $1 \mathrm{~h}$ at a concentration of $30 \mu \mathrm{g} / \mathrm{ml}$. Pre-immune rat serum was used as a control to monitor the nonspecific effect of serum on the binding of T. vaginalis to VECs. (D) Role of Tv $\alpha$-actinin 2 in cytotoxicity of T. vaginalis to VECs. T. vaginalis trophozoites were pre-incubated with anti-Tv $\alpha$-actinin 2 antibodies for $1 \mathrm{~h}$ at a concentration of $30 \mu \mathrm{g} / \mathrm{ml}$. Pre-immune rat serum was used as a control to monitor the nonspecific effect of serum on the cytotoxicity of T. vaginalis to VECs. Statistical analyses for pair-wise comparisons were performed using Student's $t$-tests to evaluate the statistical significance of these results. Differences with $p<0.05$ were considered significant. ${ }^{* *} p<0.001$.

fluorescence-labeled $\mathrm{rTv} \alpha$-actinin 2 , they were analyzed by FACS (Fig. 5A). Significant portions of the cell lines $(65 \%$ and $51 \%$ ) were labeled with fluorescent proteins in the case of VEC and RWPE-1, respectively. Binding of labeled rTv $\alpha-$ actinin 2 was also observed with a fluorescence microscope, as shown in Fig. 5B.

In addition, VECs were incubated with rTvo-actinin 2, and cell lysates were prepared for use in the western blot analysis with anti-rTv $\alpha$-actinin 2 antibodies (Fig. 5C(a)). The lysates prepared from the VECs incubated with rTv $\alpha$ actinin 2 indicated that rTv $\alpha$-actinin 2 binds to VECs (lane 3). As a loading control, the amount of human $\beta$-actin in the VEC lysates was also monitored (Fig. 5C(b)). A similar level of human $\beta$-actin was observed in each lysate sample, indicating that the absence of $\operatorname{rTv} \alpha$-actinin 2 in lane 2 of Fig. 5C(a) was not caused from a lower level of protein of human cell lysates loaded into the same lane.

\section{Determination of the Binding Domain of Tv $\alpha$-Actinin 2 to Cell Lines}

Additional experiments were performed to define the domain of rTv $\alpha$-actinin 2 involved in the adherence of 

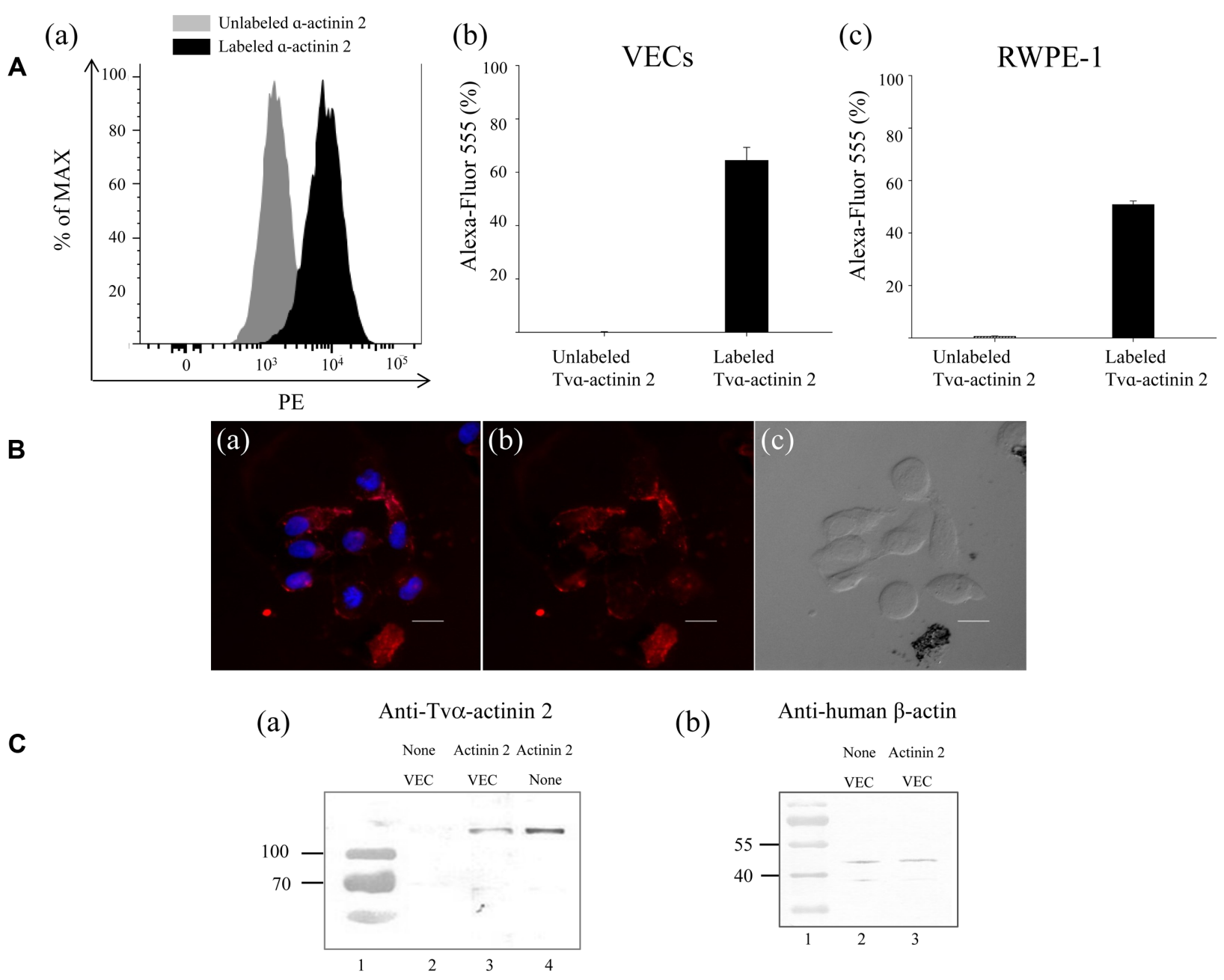

(b)

Anti-human $\beta$-actin

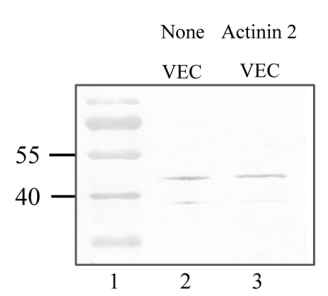

Fig. 5. Binding of $\mathrm{rTv} \alpha$-actinin 2 protein to the cell lines.

(A) Flow cytometric analysis of rTv $\alpha$-actinin 2 binding to vaginal epithelial cells (VECs) and prostate epithelial (RWPE1) cells. A histogram showing the binding of fluorescence-labeled $\alpha$-actinin 2 of $T$. vaginalis to cell lines determined by FACs. As a control, VECs were incubated with unlabeled rTv $\alpha$-actinin 2 and analyzed. (B) Labeled rTv $\alpha$-actinin 2 protein binding to VECs. (a) VECs incubated with labeled rTv $\alpha$-actinin 2 (red) merged with DAPI (blue); (b) VECs incubated with labeled rTv $\alpha$-actinin 2 (red); (c) VECs incubated with labeled rTv $\alpha$-actinin 2 under differential interference contrast. The bars represent $20 \mu \mathrm{m}$. (C) Ligand-binding immunoblotting assays. VECs $\left(2 \times 10^{5}\right)$ were incubated with $10 \mu \mathrm{g}$ of the rTv $\alpha$ actinin 2 proteins. After five washes, the cells were lysed, and analyzed by western blot assay using anti-Tv $\alpha$-actinin 2 antibodies. The same VEC lysates were analyzed for their $\beta$-actin content as a loading control by western blot assay. Lane 1: protein size marker; lane 2: VECs alone; lane 3: VECs with rTv $\alpha$-actinin 2; lane 4: rTv $\alpha$-actinin 2 (5 ng).

rTva-actinin 2 to VECs (Fig. 6). The deduced amino acid sequence of $\mathrm{Tv} \alpha$-actinin 2 revealed that this protein is composed of an N-terminal actin-binding domain, a central domain rich in $\alpha$-helices, and a C-terminal region with $\mathrm{Ca}^{2+}$-binding activity [24]. Tv $\alpha$-actinin 2 protein was separated into three parts as histidine-tagged forms (Fig. 1). Truncated $r T v \alpha$-actinin 2 proteins were determined for their cytoadherence to VECs by flow cytometry analysis (Fig. 6A). Compared with VECs without labeled protein $(5.8 \%)$, all three truncated and labeled Tv $\alpha$-actinin 2 proteins demonstrated significant binding to VECs (23 87\%). Among them, Tv $\alpha$-actinin $2 \# 1$, containing the N-terminal domain of the protein, showed the highest binding ability. Binding of these three truncated $\mathrm{rTv} \alpha$-actinin 2 proteins to VECs was observed using a fluorescence microscope (Fig. 6B). rTv $\alpha$-actinin 2 proteins were tagged with red fluorescent Alexa-Fluor 555, and then added to VECs. Only the VECs treated with $\mathrm{rTv} \alpha$-actinin $2 \# 1$ demonstrated red fluorescence, whereas VECs incubated with $\mathrm{rTv} \alpha$-actinin 2 \#2 or \#3 showed no fluorescence signal. 
A

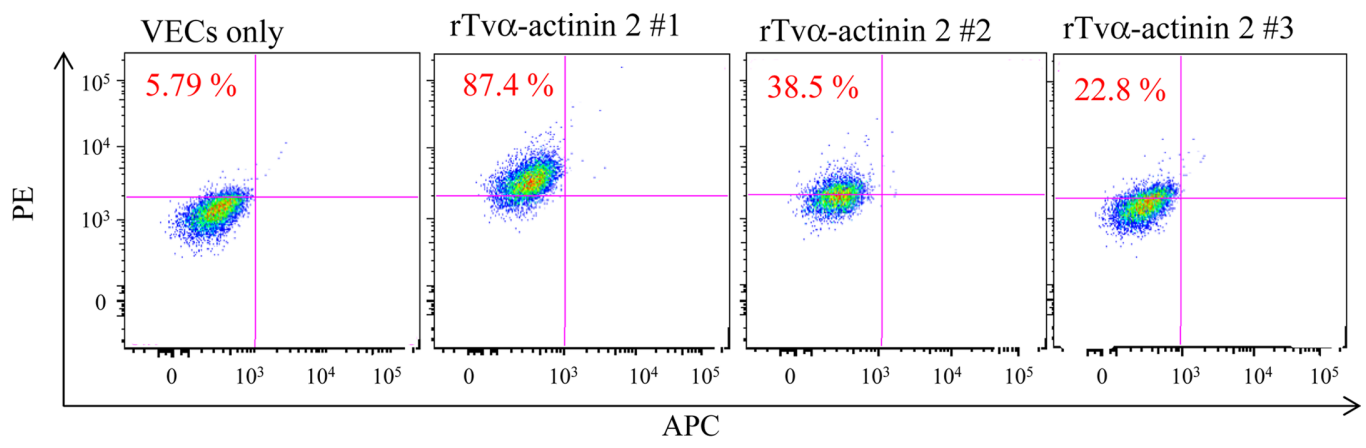

B
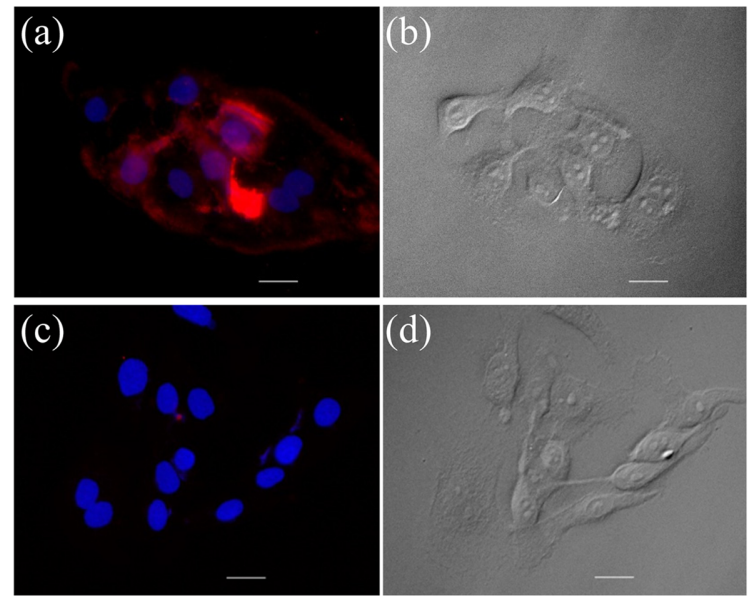

rTv $\alpha$-actinin $2 \# 2$
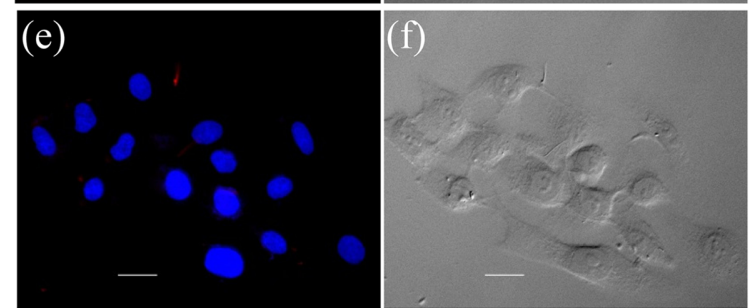

C

(a)

Anti-histidine

None \#1 \#2 \#3 \#1 \#2 \#3

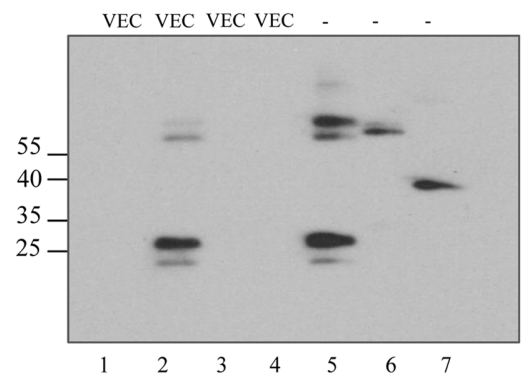

(b) Anti-human $\beta$-actin

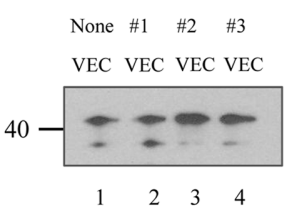

Fig. 6. Determination of the Tv $\alpha$-actinin 2 domain that binds to vaginal epithelial cells (VECs).

(A) Flow cytometric analysis of the truncated rTv $\alpha$-actinin 2 protein binding to VECs. (B) Binding of the truncated rTv $\alpha$-actinin 2 proteins to VECs. VECs with labeled, truncated rTv $\alpha$-actinin 2 (red) merged with DAPI (blue) are presented on the left side and VECs under differential interference contrast are presented on the right side. The bars represent $20 \mu \mathrm{m}$. (C) Ligand-binding immunoblotting assays. VECs $\left(2 \times 10^{5}\right)$ were incubated with $10 \mu \mathrm{g}$ of one of the three truncated $\mathrm{rTv} \alpha$-actinin 2 proteins, \#1, \#2, and \#3. After five washes, the cells were lysed, and analyzed by western blot assay using anti-histidine antibodies. The same VEC lysates were analyzed for their $\beta$-actin content as a loading control by western blot assay. Lane 1: VECs alone; lane 2: VECs with rTv $\alpha$-actinin 2 \#1; lane 3: VECs with rTv $\alpha$-actinin 2 \#2; lane 4: VECs with rTv $\alpha$-actinin 2 \#3; lanes 5-7: rTv $\alpha$-actinin $2 \# 1-\# 3$ reacted with anti-histidine antibodies (5 ng each). 
These truncated rTv $\alpha$-actinin 2 proteins were also used for ligand immunoblotting binding assays using antihistidine antibodies (Fig. 6C). Only the rTv $\alpha$-actinin $2 \# 1$ protein, containing the actin-binding domain, was detected in VEC lysates (lane 2). On the other hand, the other two rTv $\alpha$-actinin 2 proteins, \#2 and \#3, were not observed in VEC lysates (lanes 3 and 4 , respectively). Western blot analysis of human $\beta$-actin in the VEC lysates indicated that similar amounts of proteins were used in the assay. These results demonstrated that $\mathrm{rTv} \alpha$-actinin 2 binds directly to VECs via its $\mathrm{N}$-terminal region.

\section{Discussion}

After being identified as an immunogenic protein reacting to anti-T. vaginalis IgGs of female patients with trichomoniasis [24], Tv $\alpha$-actinin 2 has even been used to monitor men's serological samples for trichomoniasis exposure and risk of prostate cancer [19]. To be functional in the host interaction, this protein in the pathogen should be secreted or be present on its outermost surface. In this study, $T$. vaginalis was transformed in vitro into the amoeboid form, and Tv $\alpha$-actinin 2 was also observed near the membranes of the amoeboid form (Figs. 3B and 3C). Conversion of $T$. vaginalis into the amoebic form and the concurrent membrane localization of Tv $\alpha$-actinin 2 were observed upon incubation with VECs and RWPE-1 (Figs. 3E and $3 F$, respectively). Fractionation experiments indicated that Tvo-actinin 2 was located in the plasma membranes and secreted from T. vaginalis (Fig. 3D).

Incubation of $T$. vaginalis with VECs induced the expression of certain T. vaginalis genes, including AP-65, AP-33, and Tva-actinin 2 [27]. This is in agreement with the study of Addis et al. [25], in which the expression of Tv $\alpha$ actinin 2 increased in the amoebic form of T. vaginalis. The membrane localization and induced expression of Tv $\alpha$ actinin 2 in the amoebic form of T. vaginalis suggest its role in cytoadherence of T. vaginalis to the host cells, which may trigger an immune response or lead to death of the host cells. The role of Tv $\alpha$-actinin 2 as an immunomodulator has already been demonstrated using human epithelial cell lines and mouse dendritic cells challenged with rTv $\alpha$ actinin 2 [17]. In this study, an additional role of Tv $\alpha$ actinin 2 as an adhesin was shown by the decreased number of $T$. vaginalis that adhered to VECs and RWPE-1 in the antibody-blocking experiment (Figs. $4 \mathrm{~B}$ and $4 \mathrm{C}$, respectively). Treatment with Tv $\alpha$-actinin 2-specific antibodies resulted in the attenuated cytotoxicity to VECs (Fig. 4D), caused from the decreased binding of antibody-treated T. vaginalis. Among the identified adhesins, Tv $\alpha$-actinin 2 is the trichomonad's molecule showing immunomodulating activity.

Owing to the actin-binding domain at its N-terminus, this protein was identified as an actinin [28]. Direct interaction of Tv $\alpha$-actinin 2 with actin was observed by actin-binding assays using polymerized actin (Lee and Park, unpublished results). Thus, it is likely that Tv $\alpha$-actinin 2 becomes important under specific stages of $T$. vaginalis (i.e., upon exposure to the host cells via direct interaction with actin). A search of the T. vaginalis genome demonstrated that five Tv $\alpha$-actinin genes encode an N-terminal actin-binding domain, a C-terminal $\mathrm{Ca}^{2+}$-binding domain, and a central variable domain [29]. Among these genes, Tv $\alpha$-actinin 3 was identified to bind the iron-responsible element of Tvcp4 RNA encoding T. vaginalis cysteine proteinase 4, thus participating in post-transcriptional iron regulation. The various roles and expression of these genes should be examined.

It is possible that Tv $\alpha$-actinin 2 in the amoeboid form of T. vaginalis binds to the host epithelial cells via direct association with surface component(s) of the host cells. This study showed a direct binding of rTv $\alpha$-actinin 2 protein to the epithelial cells via flow cytometry measurement (Fig. 5A), fluorescence microscopy using the labeled Tv $\alpha$ actinin 2 proteins (Fig. 5B), and ligand immunoblotting assays (Fig. 5C). Experiments using the truncated Tv $\alpha-$ actinin 2 clearly demonstrated that the N-terminal actinbinding domain of Tva-actinin 2 plays a role in host adherence (Fig. 6).

These data indicate that Tv $\alpha$-actinin 2 plays a role in the pathogenesis of $T$. vaginalis by serving as one of the adhesins to the host cells. The $\mathrm{N}$-terminal domain containing a putative actin-binding sequence was important for its adhesion to the host epithelial cells.

\section{Acknowledgments}

This work was supported by the Basic Science Research Programs (NRF-2016R1A6A3A11934451) through the National Research Foundation (NRF) of the Ministry of Education, Science, and Technology, Korea.

\section{Conflict of Interest}

The authors have no financial conflicts of interest to declare. 


\section{References}

1. Van Der Pol B. 2007. Trichomonas vaginalis infection: the most prevalent nonviral sexually transmitted infection receives the least public health intention. Clin. Infect. Dis. 44: 23-25.

2. Lehker MW, Sweeney D. 1999. Trichomonad invasion of the mucous layer requires adhesins, mucinases, and motility. Sex. Transm. Infect. 75: 231-238.

3. Lehker MW, Alderete JF. 1992. Iron regulates growth of Trichomonas vaginalis and the expression of immunogenic trichomonad proteins. Mol. Microbiol. 6: 123-132.

4. Crouch ML, Alderete JF. 1999. Trichomonas vaginalis interactions with fibronectin and laminin. Microbiology 145: 2835-2843.

5. Tsai CD, Liu HW, Tai JH. 2002. Characterization of an ironresponsive promoter in the protozoan pathogen Trichomonas vaginalis. J. Biol. Chem. 277: 5153-5162.

6. Garcia AF, Chang TH, Benchimol M, Klumpp DJ, Lehker MW, Alderete JF. 2003. Iron and contact with host cells induces expression of adhesins on surface of Trichomonas vaginalis. Mol. Microbiol. 47: 1207-1224.

7. Peterson KM, Alderete JF. 1984. Iron uptake and increased intracellular enzyme activity follow host lactoferrin binding by Trichomonas vaginalis receptors. J. Exp. Med. 160: 398-410.

8. Lehker MW, Arroyo R, Alderete JF. 1991. The regulation of iron of synthesis of adhesins and cytoadherence levels in the protozoan Trichomonas vaginalis. J. Exp. Med. 174: 311-318.

9. Lehker MW, Chang TH, Dailey DC, Alderete JF. 1990. Specific erythrocyte binding is an additional nutrient acquisition system for Trichomonas vaginalis. J. Exp. Med. 171: 2165-2170.

10. Ardalan S, Lee BC, Garber GE. 2009. Trichomonas vaginalis: the adhesin AP51 and AP65 bind heme and hemoglobin. Exp. Parasitol. 121: 300-306.

11. Ryu JS, Choi HK, Min DY, Ha SE, Ahn MH. 2001. Effect of iron on the virulence of Trichomonas vaginalis. J. Parasitol. 87: 457-460.

12. Bastida-Corcuera FD, Okumura CY, Colocoussi A, Johnson PJ. 2005. Trichomonas vaginalis lipophosphoglycan mutants have reduced adherence and cytotoxicity to human ectocervical cells. Eukaryot. Cell 4: 1951-1958.

13. Fiori PL, Rappelli P, Addis MF. 1999. The flagellated parasite Trichomonas vaginalis: new insights into cytopathogenicity mechanisms. Microbes Infect. 1: 149-156.

14. Okumura CY, Baum LG, Johnson PJ. 2008. Galectin-1 on cervical epithelial cells is a receptor for sexually transmitted human parasite Trichomonas vaginalis. Cell. Microbiol. 10: 2078-2090.

15. Lustig G, Ryan CM, Secor WE, Johnson PJ. 2013. Trichomonas vaginalis contact-dependent cytolysis of epithelial cells. Infect. Immun. 81: 1411-1419.

16. Lee HY, Hyung S, Lee JW, Kim J, Shin MH, Ryu JS, et al. 2011. Identification of antigenic proteins in Trichomonas vaginalis. Korean J. Parasitol. 49: 79-83.

17. Lee HY, Kim J, Ryu JS, Park SJ. 2017. Trichomonas vaginalis $\alpha$-actinin 2 modulates host immune responses by inducing tolerogenic dendritic cells via IL-10 production from regulatory T cells. Korean J. Parasitol. [In Press].

18. Stark JR, Judson G, Alderete JF, Mundodi V, Kucknoor AS, Giovannucci EL, et al. 2009. Prospective study of Trichomonas vaginalis infection and prostate cancer incidence and mortality: physicians' health study. J. Natl. Cancer Inst. 101: 1406-1411.

19. Sutcliffe S, Giovannucci E, Alderete JF, Chang TH, Gaydos CA, Zenilman JM, et al. 2006. Plasma antibodies against Trichomonas vaginalis and subsequent risk of prostate cancer. Cancer Epidemiol. Biomarkers Prev. 15: 939-945.

20. Neace CJ, Alderete JF. 2013. Epitopes of the highly immunogenic Trichomonas vaginalis $\alpha$-actinin are serodiagnostic targets for both women and men. J. Clin. Microbiol. 51: 2483-2490.

21. Ryu JS, Choi HK, Min DY, Ha SE, Ahn MH. 2001. Effect of iron on the virulence of Trichomonas vaginalis. J. Parasitol. 87: 457-460.

22. Diamond LS. 1957. The establishment of various trichomonads of animals and man in axenic cultures. J. Parasitol. 43: 488-490.

23. Jin S, Joe A, Lynett J, Hani E, Sherman P, Chan VL. 2001. JlpA, a novel surface-exposed lipoprotein specific to Campylobacter jejuni, mediates adherence to host epithelial cells. Mol. Microbiol. 39: 1225-1236.

24. Addis MF, Rappelli P, Pinto De Andrade AM, Rita FM, Colombo MM, Cappuccinelli P, et al. 1999. Identification of Trichomonas vaginalis alpha-actinin as the most common immunogen recognized by sera of women exposed to the parasite. J. Infect. Dis. 180: 1727-1730.

25. Addis MF, Rappelli P, Delogu G, Carta F, Cappuccinelli P, Fiori PL. 1998. Cloning and molecular characterization of a cDNA clone coding for Trichomonas vaginalis alpha-actinin and intracellular localization of the protein. Infect. Immun. 66: 4924-4931.

26. Alderete JF. 1983. Identification of immunogenic and antibody-binding proteins on the membrane of pathogenic Trichomonas vaginalis. Infect. Immun. 40: 284-291.

27. Kucknoor AS, Mundodi V, Alderete JF. 2007. The proteins secreted by Trichomonas vaginalis and vaginal epithelial cell response to secreted and episomally expressed AP65. Cell. Microbiol. 9: 2586-2597.

28. Bricheux G, Coffe G, Pradel N, Brugerolle G. 1998. Evidence for an uncommon $\alpha$-actinin protein in Trichomonas vaginalis. Mol. Biochem. Parasitol. 95: 241-249.

29. Calla-Choque JS, Figueroa-Angulo EE, Ávila-González L, Arroyo R. 2014. $\alpha$-Actinin TvACTN3 of Trichomonas vaginalis is an RNA-binding protein that could participate in its posttranscriptional iron regulatory mechanism. Biomed. Res. Int. 2014: 4247-4267.

30. Kim SR, Kim JH, Park SJ, Lee HY, Kim YS, Kim YM, et al. 2015. Comparison between mixed lysate antigen and $\alpha$-actinin antigen in ELISA for serodiagnosis of trichomoniasis. Parasitol. Int. 64: 405-407. 